\title{
PREVALENCIA DE DIABETES MELLITUS Y SUS COMPLICACIONES EN ADULTOS MAYORES EN UN CENTRO DE REFERENCIA
}

\section{Gomezcoello Vásquez Victor ${ }^{1}$, Caza Mery ${ }^{1,2}$, Jácome Sánchez Elisa ${ }^{1 *}$.}

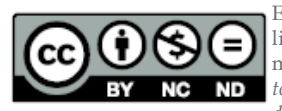

Este artículo está bajo una OPEN ACCESS o - No comercial - Sin obras derivadas 4.0 International.

1 Hospital General Enrique Garcés. Médico del servicio de Medicina Interna. Quito-Ecuador.

2 Pontificia Universidad Católica del Ecuador. Facultad de Medicina. Carrera de Medicina. Quito - Ecuador

Gomezcoello Vásquez Victor orcid.org/0000-0002-0170-8911

Caza Mery

orcid.org/0000-0001-7692-5964

Jácome Sánchez Elisa

orcid.org/0000-0002-3185-9112

*Corresponding author: Jácome-Sánchez Elisa

E-mail: elisajacome@yahoo.es

\section{Article history}

Received: 08 - Jul - 2020

Accepted: 07 - Sep - 2020

Publish: 01 - Oct - 2020

STROBE 2008 Check List statement: The

authors have real the STROBE 2008 Check Lis and the manuscript was prepared and revised according to the STROBE 2008 Checklist.

Conflict of interest: All authors declared that there are no conflicts of interest.

Financial disclosure: The authors have no financial relationships relevant to this article to disclose.

Authors' contribution: All the authors analyzed and interpreted the data, drafted the article and critically revised the article. All the authors reviewed and approved the final manuscript.

\section{Resumen}

\section{Introducción}

Una de las enfermedades crónicas no transmisibles más prevalentes del adulto mayor es la Diabetes Mellitus tipo 2 (DM2). En este grupo poblacional la DM2 se asocia a pérdida de funcionalidad, reducción de masa muscular, aumento de comorbilidades y muerte prematura, afectando significativamente la calidad de vida.

\section{Objetivo}

Establecer la prevalencia de DM2 y sus complicaciones crónicas en el adulto mayor.

\section{Pacientes y métodos}

Estudio descriptivo, transversal. Se estudiaron a 27469 pacientes adultos mayores con DM2 que acudieron a la consulta externa del Hospital General Enrique Garcés. Se investigó edad, género, etnia, zona de vivienda, nivel de instrucción, comorbilidades, tiempo de la enfermedad, medidas antropométricas, exámenes de laboratorio, complicaciones crónicas y tratamiento. Los datos fueron analizados en el programa Estadístico para las Ciencias Sociales (SPSS) v24. Se realizó un análisis descriptivo y estadística inferencial.

\section{Resultados}

El 71.13\% (19538) fueron mujeres, 66.5\% (18267) tenía educación primaria, 68\% (18679) era diabéticos más de 10 años; 85\% (23349) tenían hipertensión arterial (HTA). Las complicaciones encontradas fueron neuropatía, micro albuminuria patológica, retinopatía y pie diabético, $11.86 \%$ (3258) pacientes tuvieron una hemoglobina glicosilada ( $\mathrm{HbAlC}$ ) dentro de parámetros normales.

\section{Conclusiones}

La prevalencia de DM2 entre adultos mayores de 75 años fue del $14 \%$, y la presencia de complicaciones crónicas estuvo relacionada al mayor tiempo de evolución de la enfermedad junto a valores de HbAlC más altos.

Palabras clave: Diabetes mellitus, Diabetes mellitus tipo 2, complicaciones de la diabetes, hemoglobina glicosilada, epidemiología.
Forma de citar este artículo: Gomezcoello Vásquez V, Caza M, Jácome Sánchez E. PREVALENCIA DE DIABETES MELLITUS Y SUS COMPLICACIONES EN ADULTOS MAYORES EN UN CENTRO DE REFERENCIA. Rev Med Vozandes. 2020; 31 (2): 49 - 55 
Keywords: Diabetes mellitus, Type 2 diabetes mellitus, complications of diabetes, glycated hemoglobin, epidemiology.

\section{Abstract}

\section{PREVALENCE OF DIABETES MELLITUS AND ITS COMPLICATIONS IN OLDER ADULTS IN A REFERENCE CENTER}

\begin{abstract}
Introduction
One of the most prevalent chronic noncommunicable diseases of the older adult is type 2 Diabetes Mellitus (DM2). In this population group DM2 is associated with loss of functionality, reduced muscle mass, increased comorbidities and premature death, significantly affecting quality of life.
\end{abstract}

Objective

To establish the prevalence of DM2 and its chronic complications in the elderly.

Patients and methods

Descriptive, cross-sectional study. 27469 older adult patients with 2DM who attended the outpatient clinic of the Enrique Garcés General Hospital were studied. Age, gender, ethnicity, area of residence, level of education, comorbidities, time of illness, anthropometric measurements, laboratory tests, chronic complications, and treatment were investigated. The data was analyzed in the Statistical Program for Social Sciences (SPSS) v24. A descriptive and inferential statistical analysis was performed.

\begin{abstract}
Results
The $71.13 \%$ (19538) were women, $66.5 \%$ (18267) had a primary education, 68\% (18679) were diabetics over 10 years; $85 \%$ (23349) had high blood pressure (HT). The complications found were neuropathy, pathological micro albuminuria, retinopathy and diabetic foot, 11.86\% (3258) patients had a glycated hemoglobin ( $\mathrm{HbAlC})$ within normal parameters.

Conclusions

The prevalence of DM2 among adults over 75 years of age was 14\%, and the presence of chronic complications was related to the longer evolution time of the disease along with higher $\mathrm{HbAlC}$ values.
\end{abstract}

\section{Introducción}

De acuerdo con la Organización Mundial de la Salud (OMS) se denomina "Adulto Mayor" a cualquier persona mayor de 60 años (1). Este grupo poblacional está aumentando progresivamente, por lo tanto, las atenciones para llevar a cabo sus actividades cotidianas demanda mayor observación y atención debido al estado fisiológico propio y comorbilidades concomitantes. (2)

Durante el proceso de envejecimiento se presentan cambios fisiológicos, como el aumento del porcentaje de grasa, sobre todo la de tipo visceral, con infiltración grasa de tejidos; descenso en el agua corporal total; mayor riesgo de sobrepeso y cambios en el metabolismo de fármacos y lípidos. De igual forma a nivel del páncreas disminuye la replicación de las células beta, aumentando la apoptosis y ocasionando caída en la producción de insulina, con el consecuente aumento en la resistencia insulínica y Diabetes Mellitus tipo 2 (DM2). ${ }^{(3-5)}$

Como consecuencia de estos cambios, el adulto mayor se vuelve frágil a una variedad de enfermedades, entre ellas la DM2 que juega un rol significativo por su alto impacto en la calidad de vida. ${ }^{(2,6-8)}$

La esperanza de vida para adultos mayores de 60 años ha aumentado rápidamente en todos los países, en el Ecuador en los últimos 70 años, se ha ampliado de 48,3 años (1955) a 75,6 años (2015); para el año 2050 se espera a nivel mundial que 
existan más de 2 billones de adultos mayores, y de estos, más del $75 \%$ en países desarrollados, en nuestro país se estima que sean alrededor del 18\%. ${ }^{(9-11)}$

De igual manera, el número de adultos mayores diabéticos se triplicara, así de 12 millones en la actualidad pasará a 39 millones para el 2050. Un metaanálisis irlandés (13), demostró una prevalencia de DM2 en mayores de 65 años del 9.1\%. ${ }^{10,12)}$

La prevalencia de la DM2 creció en Latinoamérica en las dos últimas décadas ${ }^{(14)}$, y se espera que para los próximos años los patrones alimentarios y de actividad física acentúen el crecimiento epidemiológico (incidencia/prevalencia) en virtud de la mayor sobrevida de los adultos mayores. ${ }^{(5)}$

En el adulto mayor, la DM2 se asocia a pérdida de funcionalidad, reducción de masa muscular, aumento de comorbilidades (HTA, enfermedad renal, alteraciones visuales, enfermedades cardiovasculares, amputaciones) y muerte prematura. (15-17)

Según datos de la OMS (2016) en Ecuador existe una relación de 1.49:1 fallecimientos mujer/hombre en mayores de 70 años por la diabetes, en tanto que un $66 \%$ de diabéticos tiene alguna invalidez en comparación con el $29 \%$ de los no diabéticos. ${ }^{(18,19)}$

El objetivo del presente trabajo fue establecer la prevalencia de DM2 y sus complicaciones crónicas en los adultos mayores que acuden a un hospital del tercer nivel.

\section{Pacientes y Métodos}

\section{Selección de la cohorte}

Este fue un estudio de corte transversal. De 01 de enero a 31 de julio del 2018, 28.138 pacientes adultos mayores fueron atendidos en la consulta externa del Servicio de Medicina Interna del Hospital General Enrique Garcés de la ciudad de Quito - Ecuador. De estos, 27.640 pacientes participaron en el estudio. Pacientes con diagnóstico de patologías oncológicas activas o previas $(n=184)$, portadores del virus de inmunodeficiencia humana $(n=35)$, Diabéticos tipo $1(n=245)$ y portadores de ostomías $(n=34)$ fueron excluidos.

Se obtuvo el consentimiento informado de cada uno de los pacientes por escrito en el momento de la entrevista médica previa información y aceptación verbal.

\section{Diabetes mellitus tipo 2 y sus complicaciones}

\section{Tabla 1. Criterios para el diagnóstico de diabetes}

Glicemia plasmática en ayunas $\geq 126 \mathrm{mg} / \mathrm{dl}$, (Ayuno definido por al menos 8 horas) ó

Glicemia plasmática a las 2 horas posterior al test de tolerancia oral a glucosa de 75 gramos $\geq 200 \mathrm{mg} / \mathrm{dl}$, ó

Hemoglobina glicosilada $\geq 6.5 \%$, (Método que deberá ser certificado y estandarizado) ó

En pacientes con síntomas clásicos de hiperglicemia o crisis hiperglicémica una glucosa plasmática aleatoria $\geq 200 \mathrm{mg} / \mathrm{dl}$.

Tomado de. Asociación Americana de Diabetes 2020.

Los criterios diagnósticos para la DM2 usados en esta investigación se basaron en los emitidos por la Asociación Americana para la diabetes (ADA) 2020, recalcando una excepción a la hemoglobina glicosilada, la cual no es un criterio diagnóstico de acuerdo con la normativa vigente del Ministerio de Salud Pública. (15,20) (Tabla 1)

Dentro de las complicaciones macro vasculares se definió a la enfermedad cardiovascular aterosclerótica como engrosamiento y endurecimiento exclusivamente de arterias de mediano y gran calibre, donde se incluye a la cardiopatía coronaria sea infarto de miocardio, angina o insuficiencia cardíaca; enfermedad cerebrovascular como un evento vascular cerebral definitivo o un evento isquémico transitorio; y enfermedad arterial periférica como la afectación de la aterosclerosis en arterias periféricas manifestada por claudicación. ${ }^{(15,21,22)}$

En las complicaciones micro vasculares: Se definió como enfermedad renal crónica a la reducción del aclaramiento de creatinina menor de $60 \mathrm{ml} /$ $\mathrm{min} / 1.73 \mathrm{~m}^{2}$ durante al menos 3 meses; neuropatía diabética como presencia de síntomas o signos de disfunción nerviosa periférica, con algún grado de disminución de la sensibilidad en los pies, valorada con monofilamento de 10 gramos; y la retinopatía diabética como cualquier daño retiniano sea microaneurismas, exudados, neovascularización y hemorragias. (15,21,23-26)

Y por último en las complicaciones mixtas, el pie diabético se definió como un conjunto de manifestaciones neurológicas, vasculares (isquemia) e infecciosas que provoquen ulceración y pérdida de tejidos. ${ }^{(15,21,27)}$

Los datos referentes a variables demográficas, clínicas, antropométricas, complicaciones de la DM2 tanto micro y macrovasculares, tratamientos farmacológicos y exámenes de laboratorio se obtuvieron tanto de las historias clínicas como durante la entrevista directa de los pacientes.

\section{Análisis estadístico}

Los datos fueron analizados en el Programa Estadístico para las Ciencias Sociales (SPSS) v24.0. Se realizó un análisis descriptivo con medianas y frecuencias y análisis inferencial determinando asociación mediante Odds Ratio (OR).

\section{Aspectos éticos}

El estudio contó con la aprobación del Comité de Bioética de la Facultad de Medicina de la Pontificia Universidad Católica del Ecuador y por la Unidad de Docencia e Investigación del Hospital General Enrique Garcés (Quito-Ecuador), se obtuvo el consentimiento informado de todos los pacientes participantes y se siguieron las directrices STROBE para el desarrollo de esta investigación. ${ }^{(28)}$ 


\section{Resultados}

\section{Tabla 2. Análisis descriptivo de la cohorte}

\begin{tabular}{|c|c|c|}
\hline Variables clínicas & $\mathrm{n}$ & $\%$ \\
\hline \multicolumn{3}{|l|}{ Género } \\
\hline Hombre & 7930 & 28.87 \\
\hline Mujer & 19538 & 71.13 \\
\hline \multicolumn{3}{|l|}{ Edad } \\
\hline$>65$ años - 74 años & 14443 & 52.58 \\
\hline 75 - 84 años & 12034 & 43.81 \\
\hline$>85$ años & 992 & 3.61 \\
\hline \multicolumn{3}{|l|}{ E†nia } \\
\hline Mestizo & 26343 & 95.9 \\
\hline Caucásico & 137 & 0.5 \\
\hline Indígena & 852 & 3.1 \\
\hline Afroecuatoriano & 137 & 0.5 \\
\hline \multicolumn{3}{|l|}{ Zona de residencia } \\
\hline Urbana & 26041 & 94.8 \\
\hline Rural & 1428 & 5.2 \\
\hline \multicolumn{3}{|l|}{ Instrucción } \\
\hline Ninguna & 4669 & 17 \\
\hline Primaria & 18267 & 66.5 \\
\hline Secundaria & 4258 & 15.5 \\
\hline \multicolumn{3}{|l|}{ Comorbilidades } \\
\hline HTA & 23349 & 85 \\
\hline Dislipidemia & 15383 & 56 \\
\hline Lesión renal crónica & 11328 & 41 \\
\hline Hipotiroidismo clínico y subclínico & 8762 & 31 \\
\hline \multicolumn{3}{|l|}{ Tiempo de evolución de la DM2 } \\
\hline 0 a 10 años & 8919 & 32.47 \\
\hline 11 a 20 años & 10194 & 37.11 \\
\hline 21 a 30 años & 5098 & 18.56 \\
\hline 31 a 40 años & 2549 & 9.28 \\
\hline$>40$ años & 709 & 2.58 \\
\hline \multicolumn{3}{|l|}{ Peso } \\
\hline Bajo peso (IMC < 22) & 824 & 3 \\
\hline Normal (22-26) & 9889 & 36 \\
\hline Sobrepeso (27-32) & 10164 & 37 \\
\hline Obesidad (>33) & 8990 & 24 \\
\hline \multicolumn{3}{|l|}{ Perímetro abdominal } \\
\hline Mujeres $>88 \mathrm{~cm}$ & 25463 & 92.7 \\
\hline Hombres $>102 \mathrm{~cm}$ & 21151 & 77 \\
\hline \multicolumn{3}{|l|}{ Tratamiento } \\
\hline Ninguno & 274 & 1 \\
\hline Medidas higiénico-dietéticas & 2142 & 7.8 \\
\hline Antidiabéticos orales (ADO) & 1978 & 7.2 \\
\hline Insulina sola & 8488 & 30.9 \\
\hline Medidas higiénico-dietéticas + ADO & 3956 & 14.4 \\
\hline Medidas higiénico-dietéticas + Insulina & 5384 & 19.6 \\
\hline Medidas higiénico-dietéticas + Insulina + ADO & 5247 & 19.1 \\
\hline
\end{tabular}

ADO: Antidiabéticos orales; DM2: Diabetes mellitus tipo 2. Elaborado por: Los autores, 2020

\section{Características de la cohorte}

De los 27469 pacientes 19538 (71.13\%) fueron mujeres, 14443 (52.58\%) fueron menores de 74 años, la edad media de la cohorte fue de 73.3 años con una desviación estándar de 6.24 años, 26343 (95.9\%) pacientes fueron mestizos. Procedían de zonas urbanas de Quito 26041 (94.8\%) pacientes; 18267 (65.5\%) tenía instrucción primaria (Tabla 2); 27194 (99\%) estaban con tratamiento, de ellos 19228 (70\%) recibían insulina sola o combinada; y 23349 (85\%) tenían concomitantemente hipertensión arterial. En relación al tiempo desde el diagnóstico, 18550 (67\%) pacientes tuvieron DM2 más de 10 años, con un promedio de tiempo de 13.35 años y una desviación estándar de 10.32 años.

El promedio de peso en esta cohorte de pacientes fue de 67 kilos, la talla media fue de $149 \mathrm{~cm}$ y el promedio del IMC fue de $29.25 \mathrm{Kg} /$ m2, 824 (3\%) pacientes poseían un bajo peso, mientras que 10164 (37\%) tenían sobrepeso.

La glicemia basal promedio fue de $164.93 \mathrm{mg} /$ $\mathrm{dL}$ (DE +/- 136.08), el promedio de HbAlC fue de $7,75 \%$ (DE +/- 1.15), y 14866 (54.12\%) pacientes mostraron valores entre $6,5-8,5 \%$.

\section{Prevalencia de DM2 y complicaciones}

Del total de atenciones médicas realizadas en adultos mayores de 65 años, durante el periodo comprendido entre el 01 de enero a 31 de julio de 2018 correspondiente a 196.207, 27469 tuvieron DM2, lo que corresponde a una prevalencia del $14 \%$.

En cuanto a las complicaciones de la enfermedad, las más prevalentes fueron la retinopatía diabética y la micro albuminuria, con 9339 (34\%) y 11328 (41.24\%) pacientes respectivamente. (Tabla 3 )

\section{Análisis de asociación}

\section{Género-Diabetes y complicaciones:}

En el análisis de asociación, al comparar el género más diabetes e HTA, se evidenció un Odds Ratio (OR) de 0.859 (IC 95\%; 0.365-2.024; $p<0.05$ ) para los hombres, mientras que para las mujeres un OR de 1.16, de manera que los hombres diabéticos tienen menos probabilidades de padecer HTA en comparación con las mujeres.

La asociación entre género masculino y perímetro abdominal expuso un OR de 2.19 (IC 95\%; 0.8185.897; $p>0.05$ ) veces la probabilidad de presentar un perímetro normal comparado con las mujeres; No se encontró asociación con retinopatía diabética ya que la relación fue de 1.29 (IC 95\%; 0.674 a $2.471 ; p>0.05$ ) veces más riesgo para los hombres de desarrollarla, esto probablemente debido a la misma diabetes y no por la HTA. 
Tabla 3. Análisis de laboratorio y complicaciones de la cohorte

\begin{tabular}{|c|c|c|}
\hline Variables clínicas & $\mathbf{n}$ & $\%$ \\
\hline \multicolumn{3}{|l|}{ Constantes laboratoriales } \\
\hline \multicolumn{3}{|c|}{ Hemoglobina glicosilada (HB IAC) } \\
\hline$<6.5$ & 3258 & 11.86 \\
\hline $6.5-7.5$ & 8919 & 32.47 \\
\hline $7.6-8$ & 3541 & 12.89 \\
\hline $8.1-8.5$ & 2406 & 8.76 \\
\hline$>8.5$ & 9345 & 34.02 \\
\hline \multicolumn{3}{|l|}{ Glicemia Basal } \\
\hline$<70 \mathrm{mg} / \mathrm{dl}$ & 165 & 0.6 \\
\hline $71-130 \mathrm{mg} / \mathrm{dl}$ & 9477 & 34.5 \\
\hline$>131 \mathrm{mg} / \mathrm{dl}$ & 17827 & 64.9 \\
\hline \multicolumn{3}{|l|}{ Trigliceridos } \\
\hline$>150 \mathrm{mg} / \mathrm{dl}$ & 15383 & 56 \\
\hline$<150 \mathrm{mg} / \mathrm{dl}$ & 12086 & 44 \\
\hline \multicolumn{3}{|l|}{ Colesterol LDL } \\
\hline$>100$ & 16482 & 60 \\
\hline$<100$ & 10987 & 40 \\
\hline \multicolumn{3}{|l|}{ Colesterol HDL } \\
\hline$>50 \mathrm{mg} / \mathrm{dl}$ & 8515 & 31 \\
\hline$<50 \mathrm{mg} / \mathrm{dl}$ & 18954 & 69 \\
\hline \multicolumn{3}{|c|}{ Complicaciones macro vasculares de DM2 } \\
\hline IAM & 385 & 1.4 \\
\hline \multicolumn{3}{|c|}{ Complicaciones micro vasculares de DM2 } \\
\hline Retinopatía & 9339 & 34 \\
\hline Micro albuminuria & 11328 & 41.24 \\
\hline Neuropatía & 15383 & 23.2 \\
\hline \multicolumn{3}{|l|}{ Complicaciones mixtas de DM2 } \\
\hline Pie diabético & 6373 & 23 \\
\hline
\end{tabular}

ADO: Antidiabéticos orales; DM2: Diabetes mellitus tipo 2 HB IAC: Hemoglobina Glicosilada; HDL: Lipoproteínas de alta densidad LDL: Lipoproteínas de baja densidad. Elaborado por: Los autores, 2020

En cuanto al pie diabético, existe 2,5 (IC95\%; 1.120-7.338, $\mathrm{p}<0.05)$ veces más riesgo de desarrollar úlceras en los pacientes masculinos en comparación con las mujeres, probablemente debido a las actividades que ejercen los primeros. La neuropatía en pacientes varones tiene un riesgo de 1.04 (IC 95\%; 0.554-1.951, p<0.05) veces más de desarrollarse. (Tabla 4)

Tiempo de enfermedad-complicaciones DM2

La asociación entre las variables tiempo desde el diagnóstico menor a 10 años, retinopatía, micro albuminuria, úlcera del pie, neuropatía, colesterol y HbAlC demostró que los pacientes con enfermedad menor a 10 años tienen menor riesgo de complicaciones como retinopatía (OR 0.21; IC 95\%, 0.09-0.47, $\mathrm{p}<0.05$ ), micro albuminuria (OR 0.24; IC 95\%, 0.123-0.500, $p<0.05$ ), pie diabético (OR 0.66; IC 95\%, 0.23-1.92, p<0.05) y neuropatía IOR 0.22; IC 95\%, 0.11-0.43, p<0.05). El riesgo de presentar úlceras compatibles con pie diabético es de 1,65 (IC 95\%, 0.231-1.924, $p<0.05$ ) veces mayor en pacientes con más de 10 años de diagnóstico de la enfermedad. La retinopatía diabética estuvo presente con mayor frecuencia en los pacientes con más de 20 años de diagnóstico. (Tabla 5)

Hemoglobina glicosilada y complicaciones

Se demostró que mientras menor fue el valor de $\mathrm{HbAlC}$, menor fue el riesgo de presentar retinopatía diabética (OR 0.60, IC 95\%, 0.33-1.1, $\mathrm{p}<0.05$ ), micro albuminuria (OR 0.49, IC $95 \%, 0.27$ $0.88, p<0.05$ ), neuropatía (OR de 0.57, IC $95 \%$, $0.32-1.01, p<0.05)$ y pie diabético (OR 0.79, IC $95 \%, 0.31-2.0, p<0.05)$

La probabilidad de presentar pie diabético es de 1,25 (Cl 95\%, 0.31-2.00, p<0.05) veces más en pacientes con HbAlC mayor a $8 \%$.

Tabla 4. Análisis estadístico

\begin{tabular}{|lccc|}
\hline $\begin{array}{l}\text { Asociación } \\
\text { Género masculino, } \\
\text { Diabetes y Hta }\end{array}$ & 0.859 & $0.365-2.024$ & $<0.05$ \\
$\begin{array}{l}\text { Género masculino } \\
\text { y perímetro abdo- } \\
\text { minal }\end{array}$ & 2.19 & $0.818-5.897$ & $>0.05$ \\
$\begin{array}{l}\text { Género masculino } \\
\text { y retinopatía }\end{array}$ & 1.29 & $0.674-2.471$ & $>0.05$ \\
$\begin{array}{l}\text { Género masculino } \\
\text { y pie diabético } \\
\text { Género masculino } \\
\text { y neuropatía }\end{array}$ & 2.5 & $1.12-7.38$ & $<0.05$ \\
\hline
\end{tabular}

R: Odds ratio, IC: Intervalo de confianza, $\mathrm{p}$ : valor de $\mathrm{p}$ Elaborado por: Los autores, 2020

Tabla 5. Tiempo de enfermedad-complicaciones DM2

\begin{tabular}{|lll|}
\hline $\begin{array}{lll}\text { Complicaciones } \\
\text { de la DM2 }\end{array}$ & \multicolumn{2}{l|}{ Tiempo de evolución de la DM2 } \\
Retinopatía & 4.60 años (OR) & $<10$ años (OR) \\
& (IC $0.09-4.7)$ & 0.21 \\
Micro albuminuria & 4.03 & $0.09-0.47)$ \\
& (IC $0.12-0.50)$ & IC $0.123-0.500$ \\
Pie diabético & 1.65 & 0.66 \\
& (IC $0.231-1.924)$ & $(0.23-1.92)$ \\
Neuropatía & 4.4 & 0.22 \\
& (IC $0.119-0.443)$ & (IC $0.11-0.43)$ \\
\hline
\end{tabular}

R: Odds ratio, DM2: diabetes mellitus tipo2 Elaborado por: Los autores, 2020

\section{Discusión}

Se conoce ya que la fisiología del adulto mayor es diferente a la del joven, por lo que los objetivos de control tanto cardiovascular como metabólico serán distintos, de acuerdo 
a las guías ESC de HTA (2018) las cifras tensionales en adultos mayores con o sin DM2 son permisivas hasta $140 / 90 \mathrm{mmHg}$ (29), en tanto que las glicemias, en los adultos mayores sanos con pocas enfermedades crónicas concomitantes, con un estado cognitivo y funcional intacto las metas glucémicas serán una $\mathrm{HB} 1 \mathrm{Ac}$ de $7,5 \%$, mientras que aquellos con múltiples enfermedades crónicas coexistentes, deterioro cognitivo y/o dependencia funcional los objetivos glucémicos son menos estrictos, con un HBIAc entre 8.0 y $8.5 \%$, con límites entre 90 y $130 \mathrm{md} / \mathrm{dl}$. ${ }^{15,30)}$ En relación al control lipídico de acuerdo a la Sociedad Española Arterioesclerosis los valores dependerán de las patologías sobreañadidas, así en adultos mayores con DM2, HTA o un factor cardiovascular alto y con una duración de la enfermedad mayor a 10 años el colesterol LDL debe mantenerse menor a $100 \mathrm{mg} / \mathrm{dl}$, en tanto si existe un factor cardiovascular muy alto deberá ser menor de $85 \mathrm{mg} / \mathrm{dll} .{ }^{(31)}$

En nuestro estudio se encontró una prevalencia de DM2 en adultos mayores del $14 \%$, en comparación con el estudio chileno del 2018 de Peterman, et al., en donde la prevalencia bordea el $30.6 \%$, diferencia probablemente relacionada con un mayor screening en el estudio chileno ${ }^{(32)}$.

La relación mujer: hombre evidenciada en esta investigación fue de 2.46:1, similar a lo encontrado en un estudio de la provincia ecuatoriana de Bolívar, donde la proporción mujer: hombre fue de 3:1 (33), esto posiblemente debido al mayor acceso a la seguridad social por parte de los hombres, determinando una asistencia predominante de mujeres al sistema público, hallazgos que difieren con la literatura mundial donde la prevalencia es casi similar entre mujeres y hombres. ${ }^{(34)}$

Estudios internacionales de corte transversal como el de Shamshirgaran, et al. del 2017, difieren con los hallazgos de nuestro estudio, donde hemos encontrado la presencia de obesidad en pacientes con diabetes mayores de 60 años del $24.3 \%$, y de sobrepeso de $37 \%$, comparado con el 53.3 y 46.0 respectivamente, probablemente relacionada con la etnia distinta de ambas poblaciones, sin embargo, otra causa podría ser las distintas mediciones realizadas para definir el IMC en ambas cohortes. ${ }^{(33)}$

En nuestro estudio los hombres mostraron 2,19 veces más probabilidad de presentar un perímetro abdominal normal, mientras que el $90 \%$ de las mujeres lo tenían superior al normal, esto podría estar determinado ya que, en el caso de la zona urbana, las mujeres se dedican a actividades domésticas, lo que las vuelve más sedentarias y por lo tanto disminuye la actividad física, incrementando el perímetro abdominal, ítem no valorado por Shamshirgaran en su estudio.

En un estudio realizado en Reino Unido, la edad promedio de edad fue de 79 años, con un promedio de duración de la enfermedad de 9 años, en el que el 37\% de mujeres fueron obesas; la comorbilidad preponderante fue HTA, y el tratamiento más frecuentemente usado fueron antidiabéticos orales (70\%), en tanto que en nuestro estudio, la edad promedio fue de 73.3 años, el $25 \%$ de mujeres eran obesas y de igual manera la HTA fue la comorbilidad más frecuente, siendo semejante al estudio británico, sin embargo, la media de tiempo desde el diagnóstico de la enfermedad fue mayor con 14.9 años, y en relación al tratamiento en nuestro estudio el más empleado fue la insulina (30.9\%) y el menos utilizado los ADO (7.2\%) ${ }^{(35)}$
En el estudio de la Universidad de Missouri, el $90 \%$ de los pacientes tuvieron a la HTA como comorbilidad principal, mientras que en este estudio estuvo presente en el $85 \%$, dato muy similar ${ }^{(36)}$. Sin embargo, en otra investigación en el vecino país peruano, en la ciudad de Lima, la HTA estuvo presente solamente en el $55 \%$ de los pacientes, siendo la dislipidemia la comorbilidad más prevalente en el 60\%, mientras que en nuestro estudio fue menor con el $33 \%$. ${ }^{(19)}$

En el estudio francés de Le Floch, la complicación micro vascular más frecuente fue la nefropatía en un $47 \%$, difiriendo del presente ensayo, donde la complicación más frecuente fue la neuropatía periférica en el $55.67 \%$ de los casos. ${ }^{(37)}$

En un estudio Iraní, en pacientes mayores de 60 años se encontró un mayor porcentaje de complicaciones sobretodo microvasculares, hallazgos similares a los de este estudio, donde se mostró que los pacientes con más de 10 años de enfermedad tuvieron 4,6 veces más riesgo de retinopatía diabética, 4.03 para micro albuminuria positiva y 4.4 veces para presentar neuropatía diabética ${ }^{(33)}$, ambos hallazgos demuestran que la presencia de complicaciones de tipo micro vascular se relacionan de manera directamente proporcional al tiempo de diagnóstico de la enfermedad.

\section{Limitaciones}

Al ser un estudio transversal, se analizaron los datos en un momento específico de tiempo, sin otra intervención.

No se pudo realizar la escala Wifi a los pacientes con pie diabético, por el tiempo limitado de la cita médica en la consulta externa, sin embargo, esto no afectó al diagnóstico.

Al revisar la valoración oftalmológica, se encontró en los casos positivos solo el diagnóstico, sin descripción de los hallazgos del fondo de ojo y grado de lesión.

\section{Conclusiones}

En este estudio descriptivo se encontró una prevalencia de DM2 en adultos mayores del 14\%, en el que el grupo preferentemente afectado fueron las mujeres y los mayores de 74 años; la complicación más frecuentemente encontrada fue la neuropatía periférica seguida de la micro albuminuria, mientras que apenas el $23 \%$ de los pacientes tuvo pie diabético; en tanto que la comorbilidad más encontrada fue la 
hipertensión arterial y en segundo lugar la dislipidemia; el 24\% de esta cohorte fueron obesos y el tiempo desde el diagnóstico de la enfermedad en el $37 \%$ de los participantes estuvo entre los 11 a 20 años. Estos datos nos permiten definir estrategias para mejorar el screening en los adultos mayores para el inicio a tiempo de medidas preventivas para reducir el riesgo de complicaciones y permitir una mejor funcionalidad y calidad de vida a largo plazo.

\section{Agradecimientos}

Al servicio de Medicina Interna del Hospital General Enrique Garcés por permitir realizar este estudio y al Dr. Nelson Cevallos por su valiosa contribución.

\section{Referencias}

1. Instituto para la Atención de los Adultos Mayores en el Distrito Federal. Manual de cuidados generales para el adulto mayor disfuncional o dependiente. 2015. https://fiapam.org/wpcontent/uploads/2015/09/Manual_cuidados-generales.pdf

2. Dionne-Espinosa MF. El adulto mayor. Geriatria. 2004:1-23.

3. Armas M, Ballesteros M. Physiology of aging: essential study content in the training of Cuban physician. EDUMECENTRO. 2017:9(3):307-311.

4. Penny E. Geriatría y gerontología para el médico internista. Geriatría y Gerontología. 2012 doi:10.1016/j.regg.2010.02.009

5. Salech F, Jara R y Michea L. Physiological changes associated with normal aging. Rev. Med. Clin. Condes. 2012. 23(1) 19-29.

6. Lipska KJ, Krumholz H, Soones T, Lee SJ. Polypharmacy in the Aging Patient: A Review of Glycemic Control in Older Adults With Type 2 Diabetes. JAMA 2016.315(10):1034-45.

7. Genua M. Geriatría. Hospitalaria SE de F, ed. Farmacia Hospitalaria. ; 2002. www.sefh.es/ bibliotecavirtual/fhtomo2/CAP08.pdf.

8. Organización Mundial de la Salud (OMS). Informe mundial sobre el envejecimiento y la salud. J Chem Inf Model. 2015;53(9):1689-1699. doi:10.1017/CBO9781107415324.004

9. United Nations, Department of Economic and Social Affiars Population Division. World Population Ageing 2017 - Highlights.; 2017. doi:ST/ lation Ageing 201
ESA/SER.A/348

10. Cervantes-Becerra R, Villareal-Ríos EM, GarcíaRodríguez L, Vargas-Daza ER y Martínez-González L. Estado de Salud en el adulto mayor en atención primaria a partir de una valoración geriátrica integral. Aten Primaria. 2015; 47(6): 329-335. DOl: 10.1016/j.aprim.2014.07.007

11. Martínez-Pérez T, González-Aragón CM, Caste\|lón-León G y González-Aguiar B. El envejecimiento, la vejez y la calidad de vida: ¿éxito o dificultad?; Finlay. 2018. 8(1): 59-65.

12. Duda-Nyczak M. Envejecimiento en América Latina y el Caribe: Impacto en la Salud. Cepal. 2018.

13. Tracey M, McHugh S, Buckley C, Canavan R Fitzgerald A y Kearney P. The prevalence of Type 2 diabetes and related complications in a nationally representative sample of adults aged 50 and over in the Republic of Ireland. Diabet Med. 2016. 33(4):441-5. doi: 10.1111/ dme. 12845

14. Mehta R, Del Moral M y Aguilar Carlos. Epidemiología de la diabetes en el anciano.
Revista de Investigación Clínica. 2010. 62(4): 305-311.

15. American Diabetes Association. Standards of Medical Care in diabetes-2020. Diabetes Care. Volume 43, Supplement 1, January Care.
2020.

16. Affairs M, Arbor A, Israel B. Diabetes in Older Adults. 2012;35(10). doi:10.2337/dc12-1801

17. Barceló A, Rajpathak S. Incidence and prevalence of diabetes mellitus in the Americas. Rev Panam Salud Pública. 2001. 10(5):300-8.

18. Organización Mundial de la Salud. Perfiles de los países para la diabetes, 2016.2016

19. Estrada A, Hernández R, Izada L, González A, Quiñonez D y cabrera T. Clinical-epidemiologic characteristics of Type 2 Diabetes Mellitus in the polyclinic Milanés Municipality of Matanzas. Rev Méd Electrón. 2017; 39(5) 1084-1093

20. GPC Msp Ecuador. Diabetes Mellitus tipo 2 2017.

21. Ovalle-Luna D, Jiménez-Martínez I, RascónPacheco R, Gómez-Díaz R, Valdez-González A, Gamiochipi-Cano M. Prevalencia de complicaciones de la diabetes y comorbilidades asociadas en medicina familiar del Instituto Mexicano del Seguro Social. Gac Med Mex $2019 ; 1553038$

22. Sarre D, Cabrera R, Rodríguez F y Díaz E. Atherosclerotic cardiovascular disease. Review of risk scales and cardiovascular age. Med Int Méx. 2018. 34(6):910-923.

23. Gómez Ana, Arias E y Jiménez C. Insuficiencia renal crónica. Tratado de Geriatría para residentes. Sociedad Española de Geriatría y Gerontología. Capítulo 62.

24. Flores I, Cuevas Z, López R y Vásquez C. Detection of Diabetic Peripheral Neuropathy in Adults over 60 Years Old at the "Mexico BID" Health Center in Colima, Mexico. iMedPub Journals. 2018. 14 (4):1 doi: 10.3823/1399.

25. Ramírez P, Acevedo $O$ y Pedraza A. Diabetic Neuropathy: Prevalence, Risk Factors and Quality of Life in Patients from Primary Care. Arch Med Fam. 2017. 19(4):105.111

26. Guías clínicas para el manejo de la patología ocular del diabético. International council of Ophthalmology. 2017.

27. Nácher $\mathrm{V}$, Zaragozá J, Morales S, Ramírez M Sala $\vee$ y Gómez F. Valor pronóstico de la clasificación WIfl en pacientes con pie diabético. Angiología. 2017. 69(1):26-33.
28. von Elm E, Altman DG, Egger M, Pocock SJ Gotzsche PC, Vandenbroucke JP; STROBE Initiative. The Strengthening the Reporting of Observational Studies in Epidemiology (STROBE) statement: guidelines for reporting observational studies. J Clin Epidemiol. 2008 Apr;61 (4):344-9. doi: 10.1016/j.jclinepi.2007.11.008

29. Williams B, Mancia G, Spiering W, Agabiti E Azizi M, Burnier M et al. Guidelines for the management of arterial hypertension: The Task Force for the management of arterial hypertension of the European Society of Cardiology (ESC) and the European Society of Hypertension (ESH). 2018. 39(33):3021-3104.

30. Galván I, Álvarez Cl y Gómez A. Metabolic Control in the Elderly with Vulnerable and Non-vulnerable Diabetes Mellitus Type 2. Aten Fam. 2019. 26(3):95-99.

31. Ascaso J, Millán J, Hernández A, Blasco M Brea A, Díaz A. Atherogenic Dyslipidaemia 2019. Consensus document of the Atherogenic Dyslipidaemia Group of the Spanish Arteriosclerosis Society. Clin Investig Arterioscler. 2020. doi.org/10.1016/j.arteri.2019.11.004

32. Peterman F, Troncoso-Pantoja $\mathrm{Cl}$, Martínez M. et al. Asociación entre diabetes mellitus tipo2, historia familiar de diabetes y deterioro cognitivo en adultos mayores chilenos. Rev Med Chile. 2018. 146:872-881.

33. Shamshirgaran SM, Mamaghanian A, Aliasgarzadeh A, Aiminisani N, Ataie J. Age differences in diabetes-related complications and glycemic control. BMC Endocr Disord.
$2017 ;(17: 25): 1-7 . \quad$ doi:10.1 186/s12902-0172017:(17:2

34. Huelgas RG, Díez-espino J, Formiga F, Tejedor $\mathrm{JL}$. Tratamiento de la diabetes tipo 2 en el paciente anciano. Med Clin (Barc). 2012.

35. Forbes A, Murrells T, Mulnier H, Sinclair AJ. Mean $\mathrm{HbA} 1 \mathrm{c}, \mathrm{HbA} 1 \mathrm{c}$ variability, and mortality in people with diabetes aged 70 years and older: a retrospective cohort study. Lancet Diabetes Endocrinol. 2018;8587(18):1-11. doi:10.1016/S2213-8587(18)30048-2

36. Arnold S V, Lipska KJ, Wang J. Use of Intensive Glycemic Management in Older Adults with Diabetes Mellitus. J Compil Am Geriatr Soc. 2018. doi:10.1111/jgs.15335

37. Le Floch Jp, Doucet J, Verny Ch, Bauduceau B. Microvascular Complications and their Association with Survival over Five years in the GERODIAB Cohort of Elderly French Type 2 Diabetic Patients. J Diabetes Treat. 2018. DOI: 10.2901 1/2574- 7568. 000043 